\title{
Relations between concurrent hard X-ray sources in solar flares
}

\author{
M. Battaglia and A. O. Benz
}

\author{
Institute of Astronomy, ETH Zurich, 8092 Zurich, Switzerland \\ e-mail: battaglia@astro.phys.ethz.ch
}

Received 20 March 2006 / Accepted 12 June 2006

ABSTRACT

\begin{abstract}
Context. Solar flares release a large fraction of their energy into non-thermal electrons, but it is not clear where and how. Bremsstrahlung X-rays are observed from the corona and chromosphere.

Aims. We aim to characterize the acceleration process by the coronal source and its leakage toward the footpoints in the chromosphere. The relations between the sources reflect the geometry and constrict the configuration of the flare.

Methods. We studied solar flares of GOES class larger than M1 with three or more hard X-ray sources observed simultaneously in the course of the flare. The events were observed with the X-ray satellite RHESSI from February 2002 until July 2005. We used imaging spectroscopy methods to determine the spectral evolution of each source in each event. The images of all of the five events show two sources visible only at high energies (footpoints) and one source only visible at low energies (coronal or looptop source, in two cases situated over the limb).

Results. We find soft-hard-soft behavior in both, coronal source and footpoints. The coronal source is nearly always softer than the footpoints. The footpoint spectra differ significantly only in one event out of five.

Conclusions. The observations are consistent with acceleration in the coronal source and an intricate connection between the corona and chromosphere.
\end{abstract}

Key words. Sun: flares - Sun: X-rays, gamma rays - acceleration of particles

\section{Introduction}

The current understanding of solar flares leaves open fundamental questions such as: where is flare energy released, how are particles accelerated? A large part of the energy released in a solar flare is converted into energetic electrons emitting hard X-rays. Therefore, observations in X-ray wavelengths give quantitative measures of heating and particle acceleration in the flare. X-ray observations by Hoyng et al. (1981) showed hard X-ray (HXR) sources at both ends of a loop structure, commonly called footpoints. They are thick target bremsstrahlung emission produced by precipitating electrons, accelerated somewhere in the loop. Footpoints can also be seen in $\mathrm{H} \alpha$ and EUV (e.g. Gallagher et al. 2000; Fletcher et al. 2004), indicating the precipitation of flare particles and the reaction of the thermal plasma. In an event observed by Yohkoh, Masuda et al. (1994) first noted a third HXR source situated above the looptop (looptop or coronal source). Alexander \& Metcalf (1997) analyzed this event carefully, concluding that the loop top source can be best described by a thermal component and a non-thermal component which is harder than the footpoint spectrum. Petrosian et al. (2002) made an extended study of looptop sources and footpoints in Yohkohevents. They find that the spectral index of the looptop source is softer than the footpoints on the average by about 1 . The accuracy of their spectra however, was limited by the energy resolution of the Yohkoh detectors.

An important observation about the time behavior of the HXR flux has already been made in the late 1960s by Parks \& Winckler (1969) and Kane \& Anderson (1970). They found that the hardness of a spectrum changes in time and that there exists a correlation between the HXR flux and the hardness of the spectrum (soft-hard-soft or SHS). These observations were later confirmed by several authors, e.g. Benz (1977); Brown \& Loran (1985); Lin \& Schwartz (1986); Fletcher \& Hudson (2002); Hudson \& Fárník (2002). Beside the SHS pattern, a soft-hardharder (SHH) pattern has also been observed in some events (Frost \& Dennis 1971; Cliver et al. 1986; Kiplinger 1995). A quantitative study of 24 solar flares observed by RHESSI on this subject has been made by Grigis \& Benz (2004). They find that elementary flare bursts also show SHS. Battaglia et al. (2005) made a study of flares of different size, finding that events with smaller HXR flux are softer on the average and that the relation between HXR flux and spectral index at peak time of events of different size is the same as the one from several peaks of one event.

Is the SHS-behavior a feature of the acceleration mechanism as previously claimed? Or is it a transport effect produced by collisions or return currents? A further possibility could be a change in the dominating X-ray source from the coronal source (soft) to the footpoints (hard) and back to the coronal source again (soft). Thus, is the SHS-behavior nothing but a coronalfootpoint-coronal effect? The previous studies have been made using full sun spectra. To investigate the cause of the SHS, the spectra of each source must be analyzed separately. The Ramaty High Energy Solar Spectroscopic Imager (RHESSI, Lin et al. 2002) provides the possibility of making high resolution imaging spectroscopy at different locations on the sun. One can therefore study each source separately in events with several contemporaneous HXR-sources. The high energy resolution yields detailed spectra, allowing a reliable differentiation between thermal and non-thermal emission. Emslie et al. (2003) made an analysis of a very large event with 4 HXR-sources observed by RHESSI. They find a coronal source with a strong thermal component and two (at times three) footpoints in regions with opposite magnetic 
Table 1. List of analyzed events. The times give the range during which the analysis was made (times with strong emission from all three sources).

\begin{tabular}{lcr}
\hline \hline Date & Time & GOES class \\
\hline 4-Dec.-2002 & $22: 45-22: 49$ & M2.7 \\
24-Oct.-2003 & $02: 42-03: 00$ & M7.7 \\
1-Nov.-2003 & $22: 28-22: 41$ & M3.3 \\
13-Jul.-2005 & $14: 14-14: 24$ & M5.1 \\
30-Jul.-2005 & $06: 28-06: 36$ & X1.3 \\
\hline
\end{tabular}

polarity. They report that the spectral indices of the footpoints differ notably and accredit this to collisional losses by different column densities in the loop connecting the footpoints to the coronal source.

The purpose of this work is a systematic study of the relation between coronal source and footpoints in time and spectra for several well observed events. The events were carefully selected, not necessarily the largest ones, but those with informative data concerning both, thermal and non-thermal source parameters. The RHESSI data has been searched for well separated, bright events without strong pileup, situated near the limb. We present here the results for the best observed events of the first 40 months since launch.

\section{Observations, event selection and spectroscopy}

The X-ray satellite RHESSI has been observing the full sun since February 2002. Modulation of the X-ray flux by rotating grids provides image information for any region on the sun (Hurford et al. 2002). High resolution germanium detectors (energy resolution $\sim 1 \mathrm{keV}$ ) allow detailed studies of X-ray flare-spectra (Smith et al. 2002). In Sect. 2.1 we describe how events were selected. The image processing and spectral analysis methods are presented in Sect. 2.2 along with some investigations of the best choice of imaging algorithm, source regions etc.

\subsection{Event selection}

The selection was made using imaging spectroscopy quicklooks provided by the RHESSI Experimental Data Center (HEDC, Saint-Hilaire et al. 2002). Events are required to have 3 sources observed simultaneously during at least 1 minute in the course of the flare. The sources may not all be visible in the same image of a particular energy range. The search was restricted to events larger than GOES class M1 in order to have large enough count rates. Further, the three sources ought to be well separated to avoid contamination of spectra in imaging spectroscopy by other sources. For this reason, we required a minimum offset of 700 arcsec from sun center to exclude events with projection of the coronal source onto the footpoints. Events with strong particle precipitation and detector livetime (uncorrected monitor rates) below $90 \%$ were discarded. This lead to a final sample of 5 flares. Table 1 gives an overview of the selected events.

Grey-scale images of the events at $34-38 \mathrm{keV}$ (representative for emission by non-thermal electrons) are presented in Fig. 1. The 60 and $80 \%$ contours at energies 10-12 keV (dominated by thermal emission) are overplotted. The regions of interest for the spectrum calculation are given in grey. The fragmented shape of the coronal source of the Oct. 24th 2003 event can be partly accounted for by over-resolution, as the source is slightly more compact in images without detector 3 .

\subsection{Imaging spectroscopy}

In this section we discuss some technical aspects of the analysis concerning imaging spectroscopy as well as some issues that have to be considered like source separation and pileup.

\subsubsection{Imaging algorithm}

Clean, Forward-Fitting and Pixon algorithms (Hurford et al. 2002) have been tested for image reconstruction. Clean was used for the actual imaging spectroscopy for the following reasons. Forward-Fitting works fine as long as the sources in an image are equally strong, but has difficulties as soon as there are background regions that are almost as strong as a source. With the defined time and energy bins for imaging spectroscopy, this frequently occurs in high or the lowest energy bands, at any time interval, in which case Forward-Fitting produces spurious results. Pixon yields generally a better spatial separation of the sources than Clean. However, it needs more fine-tuning of the input imaging parameters to be as stable as Clean for low signal to noise ratios. In an extended series of tests, Clean turned out to be the most efficient and reliable algorithm for the automatic image generation of long time series for imaging spectroscopy.

Therefore, the Clean algorithm was applied, using detectors 3-8. Detector 2 over-resolves the sources, just increasing noise. The angular resolution without detector 3 becomes too small to separate the sources properly for all events except the one of July 13th 2005. The time bins were chosen from $12 \mathrm{~s}$ to $120 \mathrm{~s}$, depending on the source intensities, to get good images and enough counts for reliable spectra. A pseudo-logarithmic energy binning was used.

\subsubsection{Computation of spectra}

The imaging spectroscopy tools implemented in OSPEX (a further development of SPEX Schwartz 1996; Smith et al. 2002) have been used for the determination of the regions of interest (ROI) and for the calculation of the spectra. ROIs can be delimited by polygons or circles around the sources, as selected by the user. Ideally, one would select a ROI as contour in percentage of the maximum of a source. As sources move in time and often have different sizes in different energy-bands, this could not be done reasonably. Therefore, ROIs were selected as circles or polygons in each time interval, having the same size in all energies. The ROIs for the footpoints were defined at energies larger than $40 \mathrm{keV}$ such as to include all of the source emission. The ROIs of the coronal source was selected at an energy around $10 \mathrm{keV}$ likewise. The effects of this method of ROI selection are discussed in the last paragraph of this section and shown in Fig. 3.

The attained spectra were fitted with a non-thermal power law at high energies and a thermal component at low energies, where this was possible. Some of the footpoints did not have any flare emission at lower energies i.e. no measurable thermal emission. In this case only a power law was fitted to the energy range in which the flare emission was stronger than the noise level.

Figure 2 shows the spectra of the footpoints (fp 1 and 2, left and middle) and the coronal source (cs, right) for the time interval between 14:16:48-14:17:36 of the July 13th 2005 event. The fitted power-law and thermal components are also presented in Fig. 2. For each time interval and each source, we made an estimate of the noise level in the Clean images. A spectrum from a large part in the image that had not been assigned to a source 

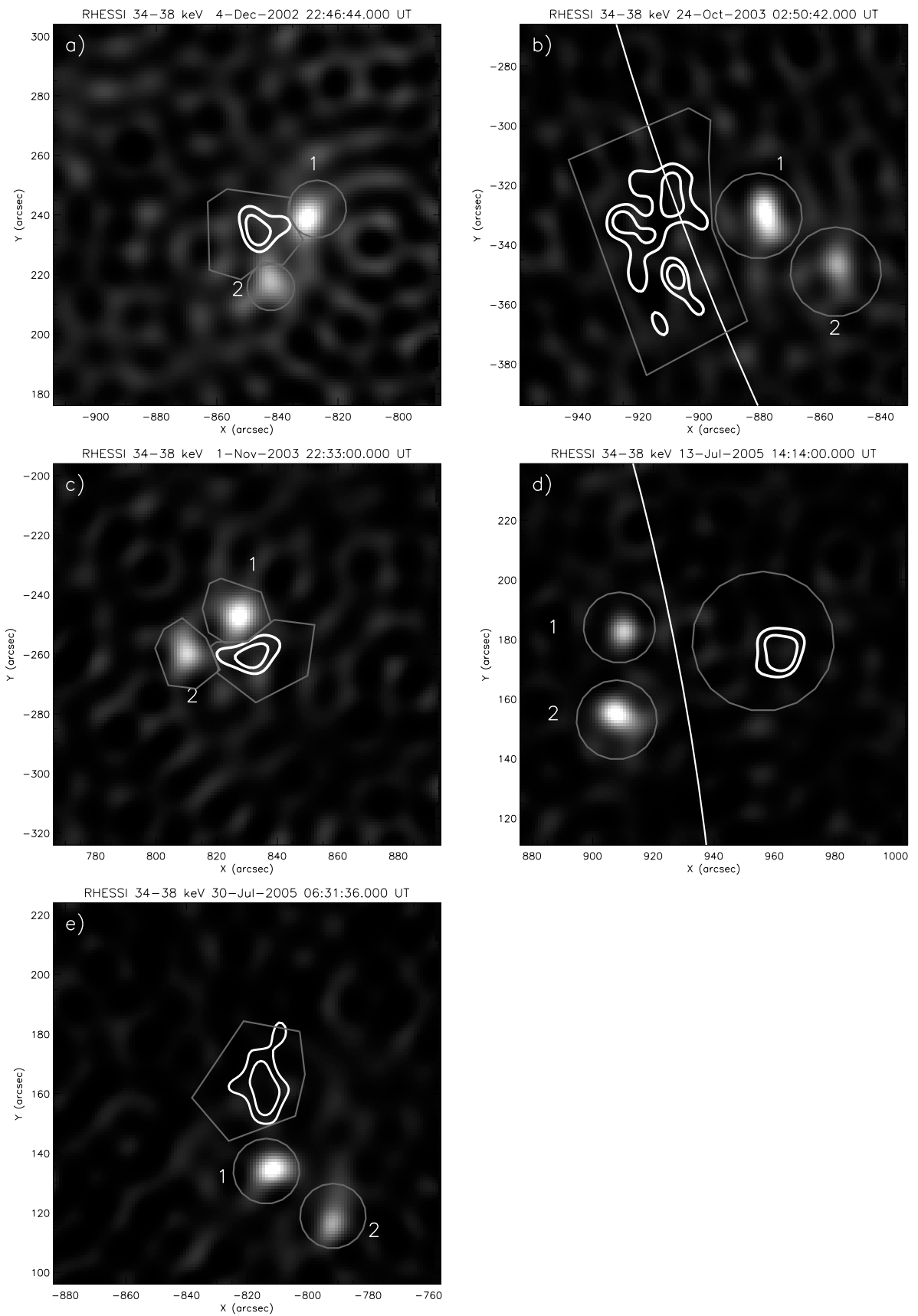

Fig. 1. Clean images (using detectors 3-8) of each event in the 34-38 keV energy range. The 60 and $80 \%$ contours at energies $10-12 \mathrm{keV}$ (white) and the regions of interest (grey) are given. The footpoints have been arbitrarily numbered (1 and 2). The solar limb is indicated where in the field of view.

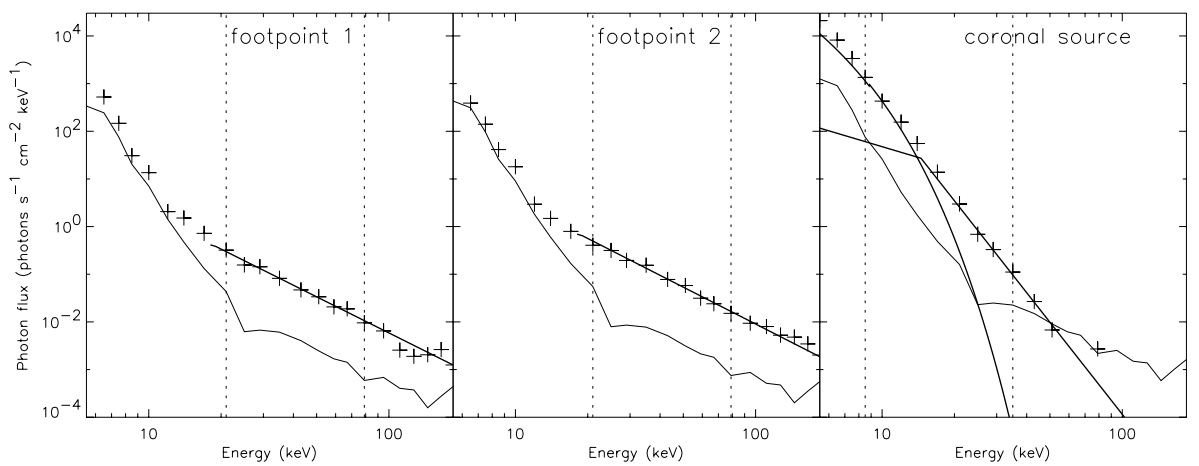

Fig. 2. Spectra of footpoints and coronal source for the time interval 14:16:48-14:17:36 of the event of July 13th 2005. The energy range for the spectral fitting is indicated by the dotted lines. The thin solid line gives an estimate for the noise level (see Sect. 2.2.2). 


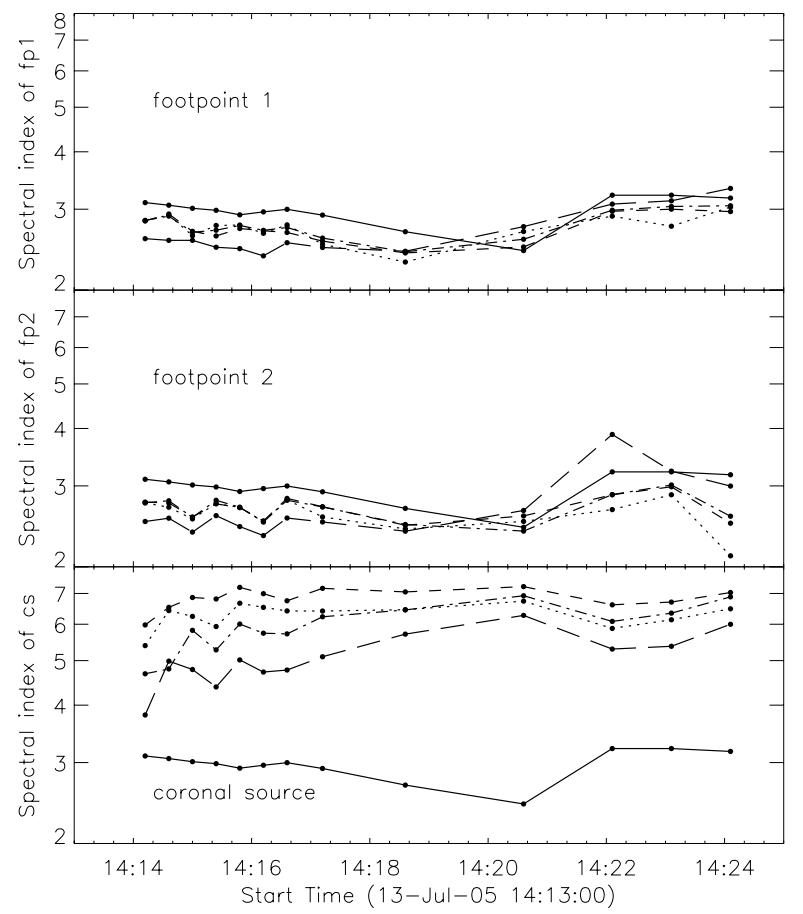

Fig. 3. Time evolution of spectral index $\gamma$ of footpoints (top and middle) and coronal source (bottom) for different choices of detectors and regions of interest. Solid: full sun; dotted: with detector 3; dot-dashed: without detector 3, same ROIs as dotted; dashed: without detector 3, different ROIs; long dashes: images with natural weighting of detectors.

has been calculated and normalized for the area of the individual source ROIs. The result is indicated by the thin lines in Fig. 2. This method will probably overestimate the actual noise, but can be used as a rough guide for the determination of the trustworthy energy intervals for the fitting (indicated by the dashed lines in Fig. 2).

The influences of the source delimitation and detector selection have been studied extensively. For the event of July 13th 2005 , tests have been made with differently defined ROIs, and with images with and without detector 3 . Finally, a series of images with natural instead of uniform detector-weighting has been compared. Natural weighting gives the same weight to each subcollimator, opposed to uniform weighting where the collimators are weighted inversely proportional to their resolution, therefore giving the finer grids more weight. Figure 3 shows the time evolution of the spectral index $\gamma$, fitted to spectra calculated for different choices of regions, detectors and weighting. The time evolution of the spectral index of full-sun spectra in the same time bins has been given for comparison. Four different cases were studied. Images with detector 3, different ROIs around the same sources, and images without detector three using the same ROIs as in the case with detector 3 , as well as images with natural detector weighting. From the time evolution of $\gamma$ one can see that the differences are small for the footpoints. The quantitative differences for the coronal source are somewhat larger. They may be used for an estimate on the error range of the spectral fittings. Note further that the non-thermal component of the full-sun spectrum is mostly due to footpoint emission. The coronal source causes a small shift toward softer spectral indices, as expected.

The qualitative behavior of the time evolution and the conclusions drawn from it do not change for the different approaches.

\subsubsection{Source separation}

The major problem encountered in the event selection was the sufficient separation of the sources. Many nice events had to be discarded because footpoints and coronal source overlap. The separation can be improved to a certain extent by optimizing parameters in the image reconstruction, but in the end one is limited by the flare morphology. Usually the footpoints are distinct and well defined. If any of the ROI's defined as described in Sect. 2.2.2 overlapped, the event was not selected. In the event of Nov. 1st 2003 (Fig. 1c) the ROIs come close, as the coronal source is embedded in a loop, visible at $10 \mathrm{keV}$, that extends nearly all the way to the footpoints. In this situation, the spectrum of the non-thermal component of the coronal source may be influenced by the emission of the footpoints. A similar case is the event of Dec. 4th 2002 (Fig. 1a). The source separation in the other three events seems clearly big enough to exclude an influence on each other.

\subsubsection{Pileup}

Although the flares in our sample are not the largest ones and events with detector livetime (uncorrected monitor rates) below $90 \%$ had been discarded in the selection, one still has to consider the possibility of pileup (Smith et al. 2002) in certain time intervals. It does not play a substantial role in the footpoints as they are observed and fitted above the energies where pileup is worst. However, the non-thermal part of the coronal source is observed at energies where pileup might cause problems. We tested the importance of pileup in our events, using the hsi_pileup_check routine. This routine calculates the corrected (counter) livetime, the effective pileup counts and the relation between corrected and uncorrected count spectra. Further, we examined images for a "ghost"-source at the position of the coronal source at higher energies. Further, we compared the time evolution of pileup flux to the time evolution of the coronal source flux at the same energy $(25 \mathrm{keV}$ for attenuator state 1$)$. The event of July 30th 2005 has attenuator state 3 throughout the observed time interval and shows no sign of significant pileup. In some of the other events (all attenuator state 1), pileup is a concern. For some times during the event of Nov. 1st and the end of Oct. 24th 2003, more than about $50 \%$ of the observed coronal HXR emission in the range between 20 and $30 \mathrm{keV}$ has to be accounted for by pileup. These times were not used in the further analysis (missing data in Fig. 4).

\section{Results}

\subsection{Soft-Hard-Soft (SHS)}

First we present the study of the SHS-behavior of individual sources.

For 4 events, the RHESSI full-sun count lightcurves in the energy-bands 3-12 keV, 25-50 keV and 50-100 keV are shown in Fig. 4. The time evolution of the fitted non-thermal flux at $35 \mathrm{keV}\left(F_{35}\right)$ and the spectral index $\gamma$ are plotted for each source. The variation in flux of the July 30th 2005 event (not shown in Fig. 4). $F_{35}$ of the footpoints correlates well with the total count flux in the $25-50$ and $50-100 \mathrm{keV}$ energy bands, indicating that the spectral fits are plausible.

In all previous observations of SHS-behavior (see Introduction) the full sun spectrum has been analyzed. As previous measurements were made at relatively high energies to avoid a contribution of the thermal component, they 

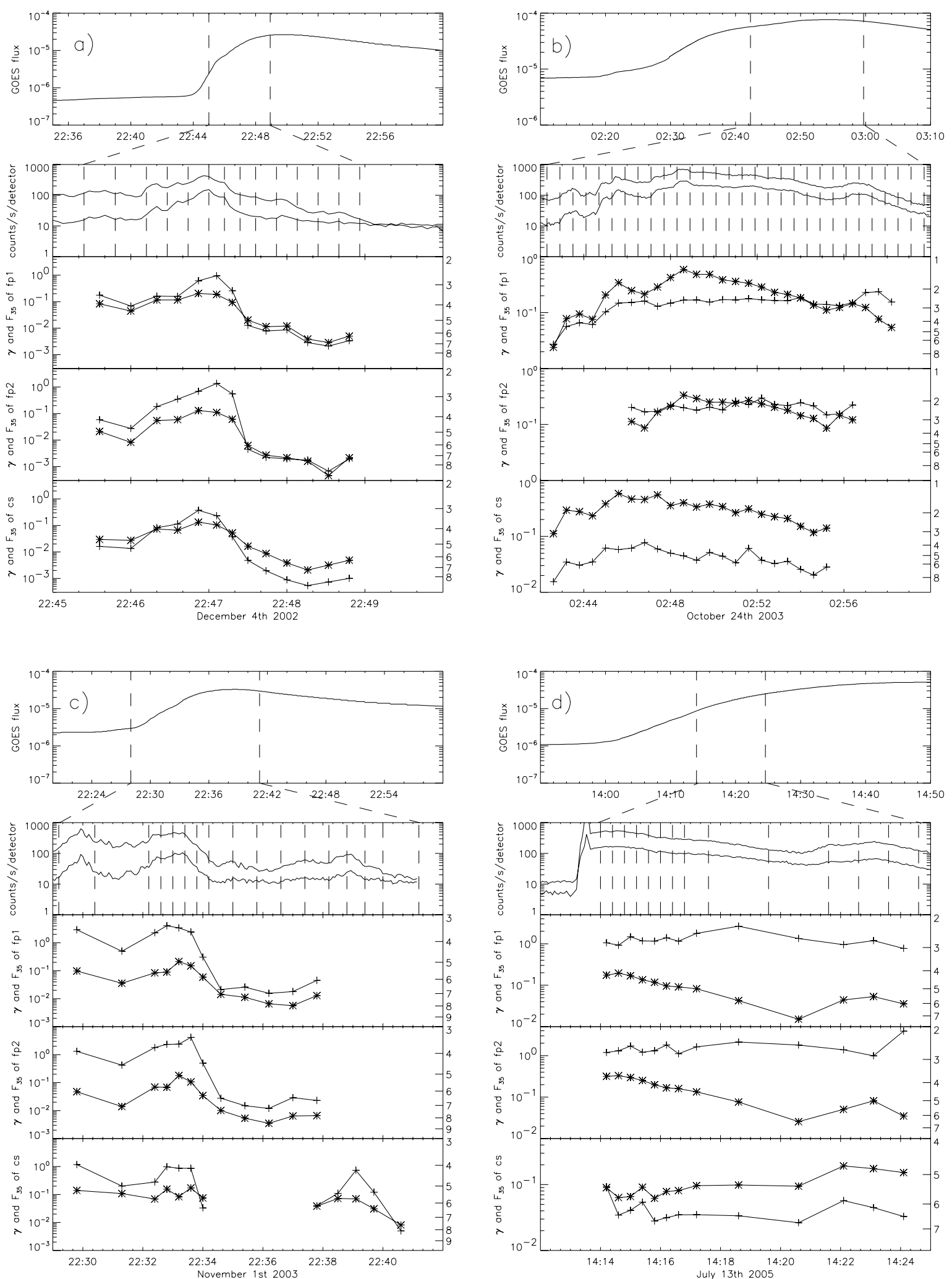

Fig. 4. Top: GOES low-channel lightcurve. The dashed lines mark the analyzed time intervals. Lower four panels: RHESSI full sun lightcurves in energy-bands $25-50 \mathrm{keV}$ and 50-100 keV, overplotted is the time binning used for imaging spectroscopy (dashed lines); time evolution of spectral index $(\gamma$, crosses $)$ and non-thermal flux at $35 \mathrm{keV}\left(F_{35}\right.$ [photons s${ }^{-1} \mathrm{~cm}^{-2} \mathrm{keV}^{-1}$ ], stars) for footpoints 1 and 2 and coronal source (bottom panel). The times between 22:34:30 and 22:37 of the Nov. 1st 2003 event had to be neglected for the coronal source because of pileup.

predominantly refer to the footpoints. For the first time, it has become possible to study the temporal evolution of the non-thermal component of the coronal source.
In all events the coronal source varies clearly according to SHS (Fig. 4). Three out of five events also show a more or less pronounced SHS-behavior in the footpoints, although there are 
times when the pattern is not very clear, or flux and spectral hardness anti-correlate. The events of Nov. 1st 2003 and Dec. 4th 2002 show a clear SHS-behavior in all sources. For Oct. 24th 2003, the variation in the flux is small without strong peaks. The event of July 13th 2005 is peculiar. There is an anti-correlation between flux (both, total count flux as well as fitted flux) and spectral hardness in the footpoints.

\subsubsection{Pivot point}

The first notion of an invariable point in solar flare spectra was made by Gan (1998). This point was termed pivot point and analyzed quantitatively for the first time by Grigis \& Benz (2004, 2005). The SHS-behavior, indicating spectral hardening at large fluxes, suggests that the non-thermal spectra at different times intersect at a fixed point in energy and flux. Grigis \& Benz (2004) noted that the intersections of all spectra in an event are within a relatively small range of energies. Its average was termed pivot energy. We applied the fitting method they describe in (2005) to determine the pivot energy for each source. An example is shown in Fig. 5. The results are given in Table 2. The physical significance of the pivot energy is not clear. However, it may be useful to describe the SHS-behavior quantitatively .

A pivot point could not be found for every source. If the variation in the flux and spectral index is small, the power-law lines are nearly parallel in $\log -\log$, and the pivot point is not well defined or does not exist. For one of the two cases where all pivot points could be determined, the pivot energy of the coronal source is higher by $3-5 \mathrm{keV}$ than the pivot energies of the footpoints (see Table 2). In the other case, the three pivot energies are equal within errors. In the two cases where only the pivot energies of the coronal sources were found, the values even exceed $20 \mathrm{keV}$. All pivot energies for both, coronal source and footpoints given in Table 2 are higher than the mean value of $9 \mathrm{keV}$ found by Grigis \& Benz (2004) for full sun spectra. The main contribution of non-thermal emission in full sun spectra originates from the footpoints. The pivot energies of the footpoints reported in Table 2 are outside the range of the half-power distribution of $6.5-12.5 \mathrm{keV}$ reported by Grigis \& Benz. However, the deviation is statistically not significant.

\subsection{Difference in spectra between sources of the same event}

Non-thermal spectra in the $20-50 \mathrm{keV}$ range can usually be well approximated by a power law (Fig. 2). The flux at a given energy and the spectral index thus characterize the spectrum of a source. In this subsection the differences between spectral indices of the coronal source and the footpoints, and between the two footpoints of each event are investigated. The mean differences in $\gamma$ time-averaged over the event are given in Table 2.

\subsubsection{Relation of coronal $\gamma$ to footpoint $\gamma$}

The coronal source is softer than both footpoints in all events at nearly all times (Fig. 4). The smallest mean difference of $0.59 \pm 0.24$ was found for the event of Nov. 1 st 2003 for which there is a possibility of source overlap. The maximum mean difference is $3.68 \pm 0.14$ for the event of July 13 th 2005 . Table 2 points out that $\gamma_{\mathrm{cs}}-\gamma_{\mathrm{fp}}$ in the 3 well separated events is remarkably larger than in the two more compact events. Note in particular, that the difference between coronal source and footpoints often differs significantly from 2 , the value expected from the
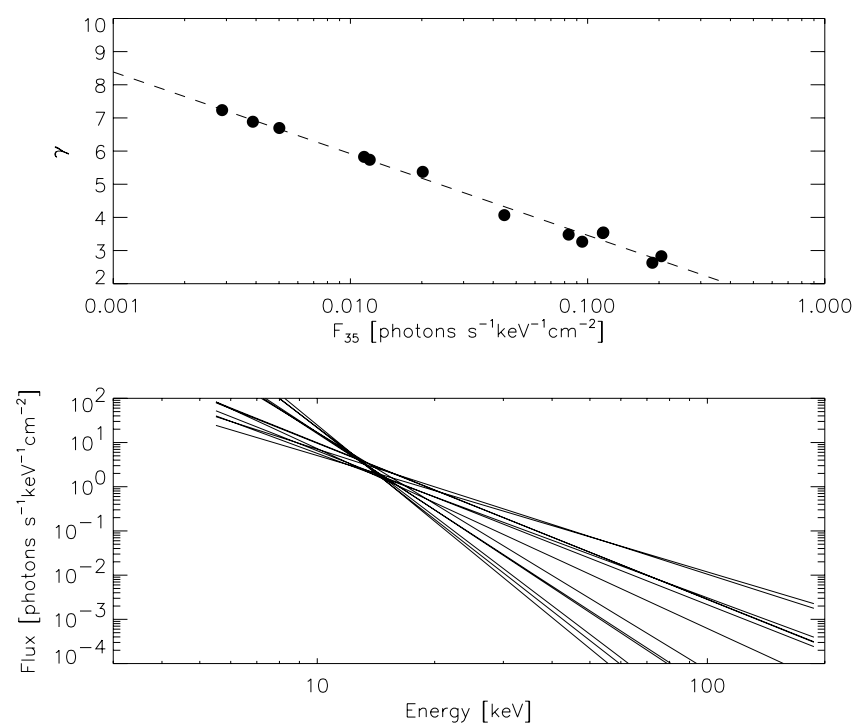

Fig. 5. Example for the determination of the pivot point (for footpoint No. 1 of Dec. 4th 2002). Top: flux and spectral index of all time intervals. The locations for the flux and spectral index in the best fitting pivot point model is shown dashed. Bottom: non-thermal power-law components for all time intervals overplotted. The crossing point agrees with the pivot point found by the above fit.

difference between thick and thin target sources. Nevertheless, the average over all mean differences is 1.82 with a standard deviation of 1.52. The weighted average and mean error are $1.98 \pm 0.42$. This finding does not agree with previous reports (Petrosian et al. 2002) based on Yohkoh data. A possible explanation for the larger value is our selection of spatially separated sources, avoiding overlap between them.

\subsubsection{Differences between footpoints}

Emslie et al. (2003) reported differences of 0.3-0.4 between the spectral indices of the two stronger footpoints in their event. For the flares analyzed here, a significant difference is found in only one out of five events, the Oct. 24th 2003 flare. For all other events, the mean difference in $\gamma_{\mathrm{fp}}$ is zero within the statistical uncertainty.

Figure 6 shows the distributions of the differences in the spectral indices of the non-thermal emission as measured in all time bins and all events. The difference between coronal source and footpoint spectral index is almost always larger than zero (Fig. 6, left). The differences between the footpoints are given in absolute values, as the footpoint numbering is arbitrary. As expected from the observations of the individual events (Fig. 4), the distribution peaks at zero.

Do the differences in spectral index change in the course of the flare? Figure 7 displays the variations through the peak (at 02:48:30) and in the decay phase. The difference between the footpoints' spectral indices does not vary within the statistical error as given by the OSPEX routine. However, the $\gamma_{\mathrm{cs}}-\gamma_{\mathrm{fp}}$ increases from peak to decay. This is caused by a considerable softening of the coronal source in this time interval (Fig. 4b).

\subsubsection{Relation between intensity and area of footpoints in the Oct. 24th 2003 event}

We compared the total flux of non-thermal photons in the $25-50 \mathrm{keV}$ range and within the $50 \%$ contour for the two 
Table 2. Mean difference in spectral index $\gamma$ between footpoints and between coronal source and footpoints for all events in which it could be determined. Pivot energy $E^{\text {piv }}$ for all sources and all events (where determinable). Temperatures derived from full sun spectra.

\begin{tabular}{|c|c|c|c|c|c|c|c|}
\hline Date & $\gamma_{\mathrm{fp} 1}-\gamma_{\mathrm{fp} 2}$ & $\gamma_{\mathrm{cs}}-\gamma_{\mathrm{fp} 1}$ & $\gamma_{\mathrm{cs}}-\gamma_{\mathrm{fp} 2}$ & $E_{\mathrm{fp} 1}^{\mathrm{piv}}$ & $E_{\mathrm{fp} 22}^{\mathrm{piv}}$ & $E_{\mathrm{cs}}^{\mathrm{piv}}$ & Temperature (MK) \\
\hline 4-Dec.-2002 & $-0.53 \pm 0.20$ & $1.22 \pm 0.20$ & $0.68 \pm 0.15$ & $13.74 \pm 0.41$ & $14.97 \pm 0.63$ & $18.12 \pm 0.25$ & $18.30 \pm 1.68$ \\
\hline 24-Oct & $0.33 \pm 0.04$ & $2.43 \pm$ & $3.07 \pm$ & - & - & $22.74 \pm 2.99$ & 0.05 \\
\hline 1-Nov.-2003 & $-0.095 \pm 0.093$ & $0.72 \pm 0.16$ & $0.59 \pm 0.24$ & $14.68 \pm 1.14$ & $14.00 \pm 1.33$ & $15.90 \pm 2.36$ & 20.65 \\
\hline 13-Jul.-2005 & $0.13 \pm 0.07$ & $3.55 \pm 0.13$ & $3.68 \pm 0.14$ & - & - & - & $24.95 \pm 1.07$ \\
\hline 30-Jul.-2005 & $0.13 \pm 0.07$ & $1.15 \pm 0.38$ & $1.12 \pm 0.41$ & _- & _- & $23.92 \pm 2.37$ & $24.85 \pm 0.31$ \\
\hline
\end{tabular}
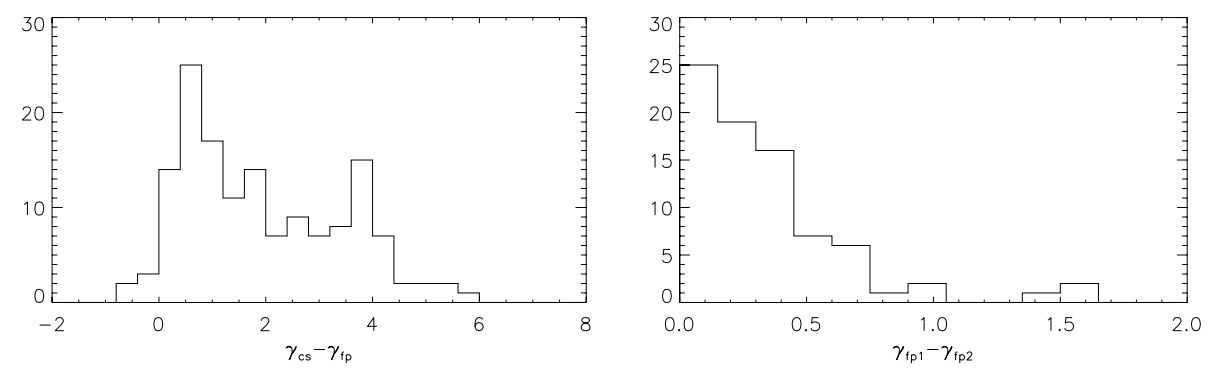

Fig. 6. Distributions of the differences in spectral index $(\gamma)$ between coronal source and both footpoints of all events at all time bins $(l e f t)$ and between footpoints only (right). For the differences between footpoints, absolute values are shown.

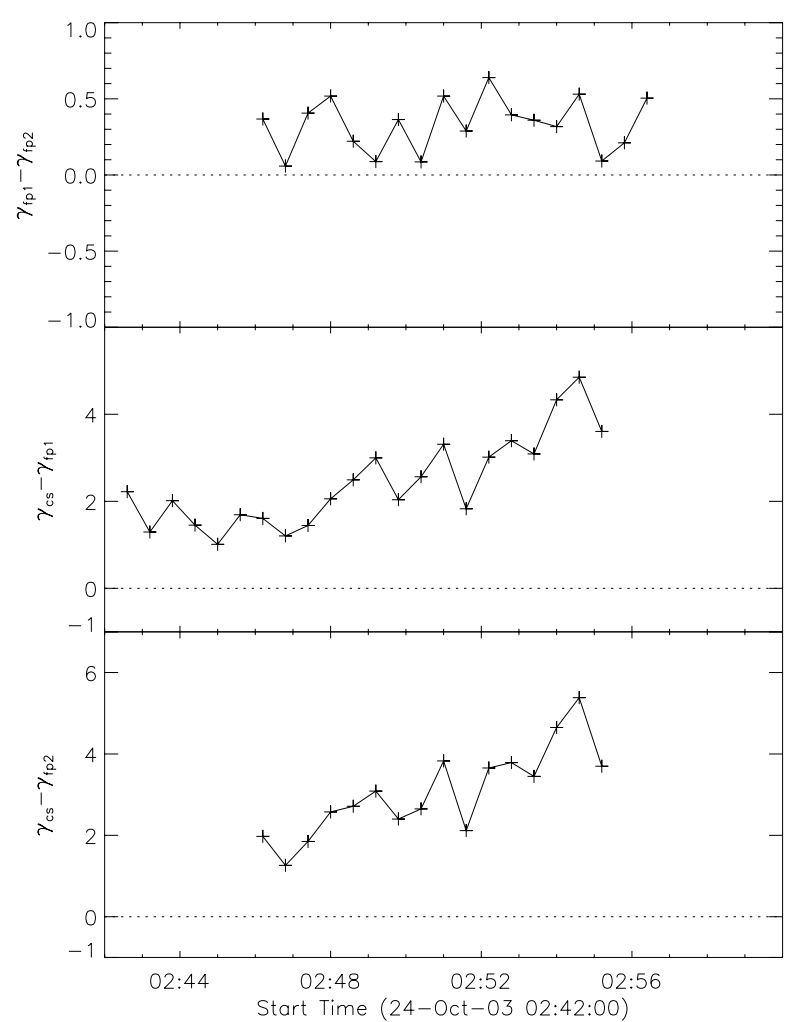

Fig. 7. Time evolution of the difference between spectral indices of the three sources for the event of Oct. 24th 2003.

footpoints of the Oct. 24th 2003 event for the times where they were best observed.

We determined the footpoint flux from cleaned images using detectors 3-8 and with a pixel size of 0.5 in the $25-50 \mathrm{keV}$ range within the $50 \%$ contour $\left(F_{50 \%}\right)$ and define the intensity $f_{50 \%}$ as ratio of flux divided by the area $\left(A_{50 \%}\right)$ of the contour,

$I_{50 \%}=\frac{F_{50 \%}}{A_{50 \%}}$, where the area of the clean beam has been subtracted. Figure 8 shows the time evolution of $F_{50 \%}$, the area $A_{50 \%}$ and the intensity $I_{50 \%}$.

The total flux $F_{50 \%}$ correlates with the full sun count flux. Further, it correlates with the fitted flux $F_{35}$ (comp. Fig. 4), validating the applied methods. The softer footpoint, No. 1, is always brighter than footpoint No. 2. and is larger in area. The harder footpoint (No. 2) has the higher intensity for most of the observed time.

\subsubsection{Thermal emission of footpoints}

The coronal source dominates at low energies in all selected events. Images at low energies often do not show strong emission at the position of the footpoints. Sometimes no fit to the thermal component of the footpoints was possible. As demonstrated in Fig. 2, the noise level at low energies (few keV up to $10 \mathrm{keV}$ ) reaches about a tenth of the emission of the coronal source.

A rough overview on all times of all events yields the following statistics on the thermal emission of the footpoints:

- One footpoint with measurable thermal emission during more than $50 \%$ of the time (fp No. 1 of the Dec. 4th 2002 event).

- Two footpoints with no measurable thermal emission at all times (fp No. 2 of the July 30th 2005 event and fp No. 2 of Oct. 24th 2003).

- All other footpoints show thermal emission in the spectrum for $20 \%$ of the time on the average.

A formal fitting has been performed at the defined regions of interest for the undetected thermal footpoint emission in Fig. 2. The result corresponds to the uncertainty level and yields an upper limit for the thermal footpoint emission. The relations between the emission measure of the coronal source and the footpoints are

$$
\frac{E M_{\mathrm{fp} 1}}{E M_{\mathrm{cs}}}<0.2
$$




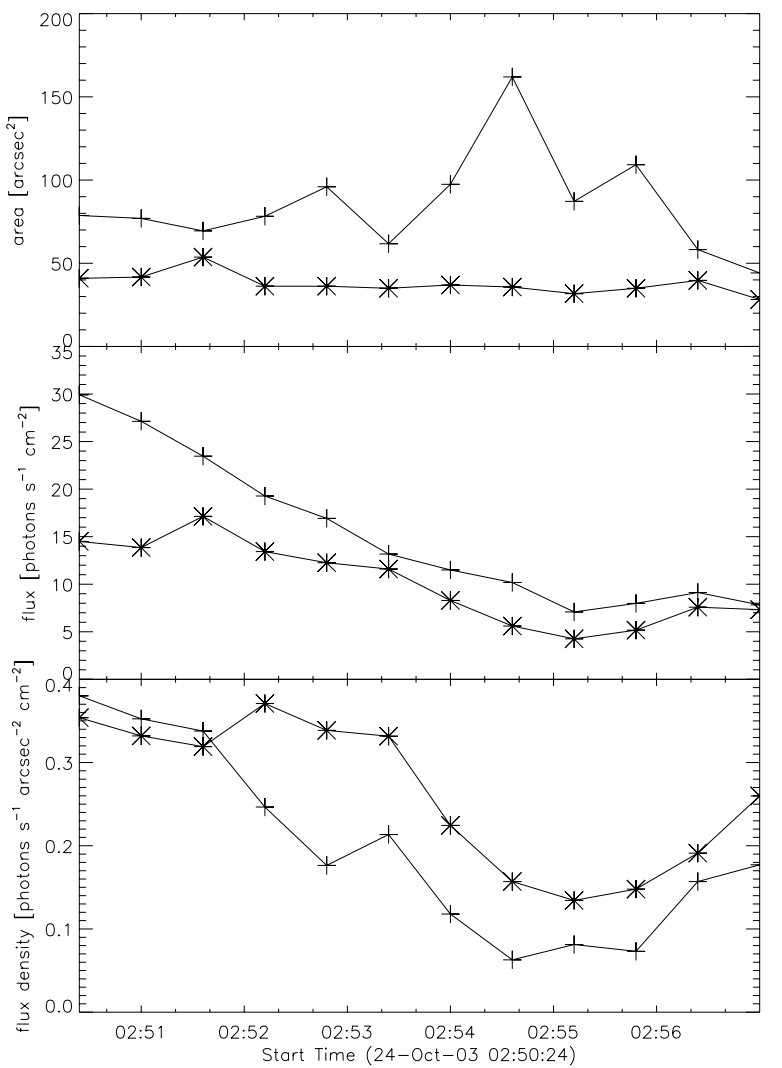

Fig. 8. Time evolution of area of $50 \%$ contour in the $25-50 \mathrm{keV}$ image, flux within contour in photons $\mathrm{s}^{-1} \mathrm{~cm}^{-2}$ and the intensity (flux/area) of footpoints of Oct. 24th 2003 event. Crosses show footpoint No. 1, asterisks indicate footpoint No. 2 as numbered in Fig. 1.

and

$\frac{E M_{\mathrm{fp} 2}}{E M_{\mathrm{cs}}}<0.1$

The fact that almost all of the footpoints studied here have a thermal spectrum at some time and the well-known fact that flare observations at EUV wavelengths show thermal emission from the footpoints suggest that there may well be thermal emission from footpoints all the time. In soft X-ray observations by Yohkoh/SXT, such emission has been reported e.g. by McTiernan et al. (1993) and Hudson et al. (1994). However, RHESSI can only observe it if the emission measure is at least $10 \%$ of the coronal source and at a temperature of several million Kelvin. Therefore, the thermal emission measured in full sun spectra is predominantly emission of the coronal source. Table 2 gives the average temperatures as fitted to full sun spectra. They are representative for the temperature of the coronal source.

\section{Discussion}

\subsection{Coronal source shows SHS-behavior}

Although previously reported in the literature, the existence of a non-thermal component in the coronal source is not trivial. As the thermal component is strong and any non-thermal emission very soft, the latter is just an extension at a much lower flux (see Fig. 2c). We have tested the possibility of flux pileup contributing to the range of energies where the non-thermal component was fitted (Sect. 2.2.4). These tests show that the observed HXR tail cannot be caused just by pileup but that there is significant HXR source emission. We cannot exclude in all cases, however, that the extension cannot be fitted equally well with a second thermal component at a much higher temperature. In cases like Fig. 2c, the fit with two thermal components has a higher $\chi$-square and is therefore less likely. We will thus continue to refer to the high-energy extension as non-thermal.

It is a remarkable result that for 5 out of 5 events, the timeevolution of the spectral index $\gamma$ of the coronal sources shows SHS-behavior. The event of July 13th 2005 is noteworthy, showing SHS-behavior in the coronal source, but not in the footpoints (Fig. 4d). The SHS-behavior of the coronal source would not be expected if SHS was just a transport effect such as Coulomb collisions or an electric field. Filtering of low energy electrons in the loop by collisions would not have an effect on the coronal source from where the particles may have originated. An induced electric field due to the return current $E=\eta j^{\text {ret }}$ (where $\eta$ is the electric resistivity), reflecting low energy particles from the loop back upwards would even lead to a softer spectrum in the coronal source, i.e. an anti-correlation between flux and spectral hardness.

Although the notion of a pivot point was introduced by Grigis \& Benz (2004) as a convenient and quantitative characterization of the SHS-behavior, Table 2 suggests a possible physical significance of the pivot energy: $E_{\mathrm{cs}}^{\mathrm{piv}}$ seems to increase with the temperature of the coronal source. The significance needs to be confirmed by a larger sample. Another hint on a possible physical relevance is the value of the pivot energy. In the flares in which it could be determined, the pivot energy of the coronal source is at the energy (within the error range) where the spectra of the thermal and non-thermal components intersect (Fig. 2c) or higher. Table 2 also shows that the pivot energy is an order of magnitude higher than the mean thermal energy. A deviation from a Maxwellian energy distribution or from isothermal homogeneity would be necessary to interpret the pivot energy as the starting point for the non-thermal acceleration. We do not consider it impossible, but highly speculative.

\subsection{Soft-hard-soft behavior of the full sun}

The soft-hard-soft (sometimes soft-hard-harder) behavior of solar flares has been extensively studied in full sun observations (see Introduction). As the coronal source usually dominates in the early phase of an event and remaines luminous longest, but at peak time the non-thermal part of full-sun spectra is dominated by the footpoints (e.g. Fig. 3), the reported SHS results of full sun observations need to be tested for the possibility of spatial changes dominating temporal changes.

We have compared the time evolution of the non-thermal flux $F_{35}$ of the coronal source and of the combined footpoints to investigate the influence of a change in predominance from the coronal source emission to footpoint emission and back to coronal emission (coronal-footpoint-coronal) on the SHS feature. Figure 9 shows the only event where an indication of such an effect could be found. The footpoint emission is weaker than the coronal source in the beginning, exceeds the coronal emission when the spectral index is hardest and decreases below the level of coronal emission afterwards. The (negative) spectral index of the full sun correlates with both the flux of footpoints and the flux of the coronal source. It continues to correlate with the coronal source when the footpoints vanish. We conclude that a coronal-footpoint-coronal effect may enhance the SHS feature in full sun observations, but does not cause it. Therefore, the SHSbehavior must be a property of the sources themselves. 


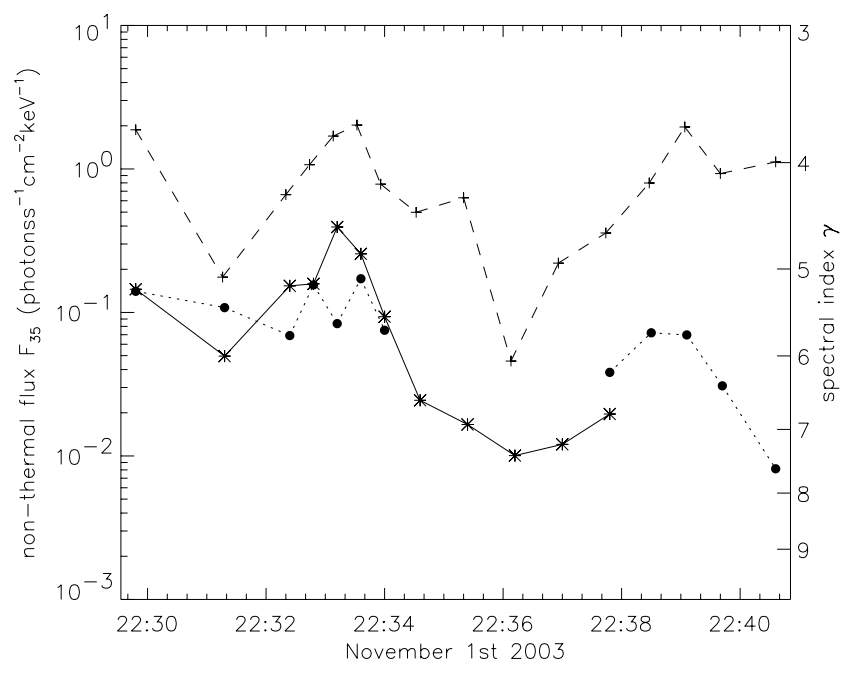

Fig. 9. Non-thermal flux $F_{35}$ fitted to the spectrum of the coronal source (dotted line) compared to summed flux of both footpoints (solid line) of the event of Nov. 1st 2003. The dashed line gives the spectral index of the full sun spectra.

\subsection{Differences in spectra}

\subsubsection{Difference between coronal source and footpoints}

Assuming an electron power-law distribution for the electron energy $E$ of the form

$F(E)=A E^{-\delta}$

producing thin-target bremsstrahlung-emission in the coronal source, the observed photon-spectrum at photon energies $\varepsilon$ is

$I_{\text {thin }}(\varepsilon) \sim \varepsilon^{-(\delta+1)}$

with spectral index $\gamma_{\text {thin }}=\delta+1$. Reaching the chromosphere, the accelerated electrons will be fully stopped, producing thicktarget bremsstrahlung with a photon-spectrum

$I_{\text {thick }}(\varepsilon) \sim \varepsilon^{-(\delta-1)}$

having a spectral index $\gamma_{\text {thick }}=\delta-1$ (Tandberg-Hanssen \& Emslie 1988). In such a simple scenario one would therefore expect a difference in the photon spectral index $\gamma_{\text {thin }}-\gamma_{\text {thick }}=2$ between the coronal source and the footpoints. Indeed we find always a difference between the spectral index of the coronal source and the footpoints. In 2 events out of 5, the difference is considerably larger than 2 . This result excludes a scenario in which the same electron beam first produces thin target emission in the corona, then thick target emission in the chromosphere. Different particle populations seem to be involved or a filter mechanism may operate causing low-energetic electrons to preferentially lose energy before they reach the chromosphere and therefore hardening the spectrum. Candidates for such a transport effect are collisions and the electric field of the return current.

The 3 events in which the difference is smaller than two include those two with small source separation. The similarities in the spectra can therefore be partly accounted for by a situation that is between the assumed ideal thick and thick targets. This may be the case when electrons substantially lose energy before reaching the chromosphere.

\subsubsection{Difference between footpoints}

The difference between the footpoint spectral indices is only significant in one event out of five (Oct. 24th 2003). The larger and more luminous footpoint is softer. The opposite is the case in the flare of July 23rd 2003, analyzed by Emslie et al. (2004).

\section{Conclusions}

A selection of five RHESSI events with three concurrent X-ray sources (coronal source and two footpoints) has been studied regarding the spectral relations between the sources. All spectra can be fitted with a non-thermal component having a powerlaw photon distribution. Although no low-energy soft X-ray observations are available for comparison, we believe that they probably are examples for Masuda-type sources (Masuda 1994), but our looptop sources are generally softer than the one found by Masuda. Therefore, such events are easier to detect with RHESSI than they were with Yohkoh and are more frequent than inferred previously. In addition, all coronal sources and some of the footpoints at times show a thermal component. The major results are:

- All coronal sources evolve according to the same time evolution in spectral hardness. The higher the flux, the harder (smaller $\gamma$ ) the non-thermal component. This soft-hard-soft pattern correlates with the non-thermal flux without measurable delay. Transport effects such as collisions or an induced electric field cannot cause SHS in the coronal source.

- As the emission of the footpoints often dominates at $35 \mathrm{keV}$, it is not surprising that the pattern, previously reported for full sun observations, is also found in the footpoints of three out of 5 events. Imaging spectroscopy suggests that SHS is a feature of all sources, and thus possibly of the accelerator itself.

- SHS in full sun observations cannot be explained by a change of the dominant source (softer coronal source present at all times plus a hard footpoint source with time-varying intensity). If SHS was caused by such an effect, neither source would display it individually.

- The time averaged difference in spectral index between the non-thermal coronal and footpoint emission is not 2 for individual events, as would be the case if the difference was simply caused by thin and thick target bremsstrahlung, respectively. Smaller differences in $\gamma$ may be explained by an intermediate situation between the two extremes. The plasma of the coronal source could act as thick target for low energetic electrons and as thin target for higher electron energies. The cases with $\gamma>2$ require a filter effect in the propagation preferentially reducing the distribution at lower energies. Such a filter may be collisions or an electric field.

- The pivot energy, characterizing the SHS-behavior of the nonthermal emission, is at the energy where the distribution of the thermal and non-thermal components balance in half of the cases. In the other half, the pivot energy is higher than this point.

- The pivot energy at the footpoints is significantly lower in all cases (14-15 keV for the footpoints vs. $16-23 \mathrm{keV}$ for the coronal source). Such a difference suggests a filter acting during particle propagation to the footpoints, reducing lower energies more than higher energies.

- In one out of 5 events the two footpoints have significantly different spectral indices, $\Delta \gamma=0.33 \pm 0.04$. The difference is constant during the event, although the spectral indices vary in time. Again, an energy filter during propagation seems to be at work, differing in one flare for the two legs of the loop. 
- The photon flux at energies below about $15 \mathrm{keV}$ is dominated by thermal emission. Most of this emission originates from the coronal source. If its temperature correlates with the pivot energy it may hint at a physical significance of the pivot energy for the acceleration process, but needs further investigation.

- As pointed out before from Yohkoh/SXT observations, the thermal emission from the coronal source often significantly exceeds the thermal emission of the footpoints, which is detectable in some events and at some times.

This analysis has shown that the non-thermal X-ray emission in coronal sources cannot only be detected by RHESSI, but can also be studied in time. As the coronal source is directly related to flare energy release, this opens the possibility of further investigating the enigmatic acceleration process of electrons. The temporal and spectral relation of the coronal source to the footpoints suggests an intricate connection between corona and chromosphere. While a comprehensive interpretation of our results in terms of particle propagation and thermal conduction is beyond the scope of this study, the idea that flare energy release and particle acceleration are closely related to the coronal source is supported by our results.

Acknowledgements. RHESSI data analysis at ETH Zürich is supported by ETH grant TH-1/04-2 and the Swiss National Science Foundation (grant 20-105366). Much of this work relied on the RHESSI Experimental Data Center (HEDC), developed under ETH Zürich grant TH-W1/99-2. We thank Säm Krucker, Paolo Grigis, Gordon Hurford and the unknown referee for helpful comments and discussions, and André Csillaghy and Kim Tolbert for help with the software.

\section{References}

Alexander, D., \& Metcalf, T. R. 1997, ApJ, 489, 442

Battaglia, M., Grigis, P. C., \& Benz, A. O. 2005, A\&A, 439, 737

Benz, A. O. 1977, ApJ, 211, 270

Brown, J. C., \& Loran, J. M. 1985, MNRAS, 212, 245

Cliver, E. W., Dennis, B. R., Kiplinger, A. L., et al. 1986, ApJ, 305, 920

Emslie, G. A., Kontar, E. P., Krucker, S., \& Lin, R. P. 2003 ApJ, 595, L107

Fletcher, L., \& Hudson, H. S. 2002, Sol. Phys., 210, 307

Fletcher, L., Pollock, J. A., \& Potts, H. E. 2004, Sol. Phys., 222, 279

Frost, K. J., \& Dennis, B. R. 1971, ApJ, 165, 655

Gallagher, P. T., Williams, D. R., Phillips, K. J. H., et al. 2000, Sol. Phys., 195, 367

Grigis, P. C., \& Benz, A. O. 2004, A\&A, 426, 1093

Grigis, P. C., \& Benz, A. O. 2005, A\&A, 434, 1173

Hoyng, P., Duijveman, A., Machado, M. E., et al. 1981, ApJ, 246, L155

Hudson, H. S., \& Fárník, F. 2002, Solar Variability: From Core to Outer Frontiers, ESA SP-505, 261

Hudson, H. S., Strong, K. T., Dennis, B. R., et al. 1994, ApJ, 422, L25

Hurford, G. J., Schmahl, E. J., Schwartz, R. A., et al. 2002, Sol. Phys., 210, 61

Gan, W. Q. 1998, Ap\&SS, 260, 515

Kane, S. R., \& Anderson, K. A. 1970, ApJ, 162, 1003

Kiplinger, A. L. 1995, ApJ, 453, 973

Lin, R. P., \& Schwartz, R. A. 1986, ApJ, 312, 462

Lin, R. P., Dennis, B. R., Hurford, G. J., et al. 2002, Sol. Phys., 210, 3

Masuda, S. , Kosugi, T., Hara, H., et al. 1994, Nature, 371, 495

McTiernan, J. M., Kane, S. R., Loran, J. M., et al. 1993, ApJ, 416, L91

Parks, G. K., \& Winckler, J. R. 1969, ApJ, 155, 117

Petrosian, V., Donaghy, T. Q., \& McTiernan, J. M. 2002, ApJ, 569, 459

Saint-Hilaire, P., von Praun, C., Stolte, E., et al. 2002, Sol. Phys., 210, 143

Schwartz, R. A. 1996, Compton Gamma Ray Observatory Phase 4 Gues Investigator Program: Solar Flare Hard X-ray Spectroscopy, Technical Report, NASA Goddard Space Flight Center

Smith, D. M., Lin, R. P., Turin, P., et al. 2002, Sol. Phys., 210, 33

Tandberg-Hanssen, E., \& Emslie, A. G. 1988, The Physics of Solar Flares, Cambridge Astrophysics Series 